[Highlight]

\title{
单位点催化剂的简便构筑方法、原理及性能研究
}

刘忠范

北京大学化学与分子工程学院, 北京 100871

\section{The Simplest Construction of Single-Site Catalysts for Efficient Energy Conversion}

\section{LIU Zhongfan}

College of Chemistry and Molecular Engineering, Peking University, Beijing 100871, P. R. China. Email: zfliu@pku.edu.cn.

Published online: April 24, 2019.
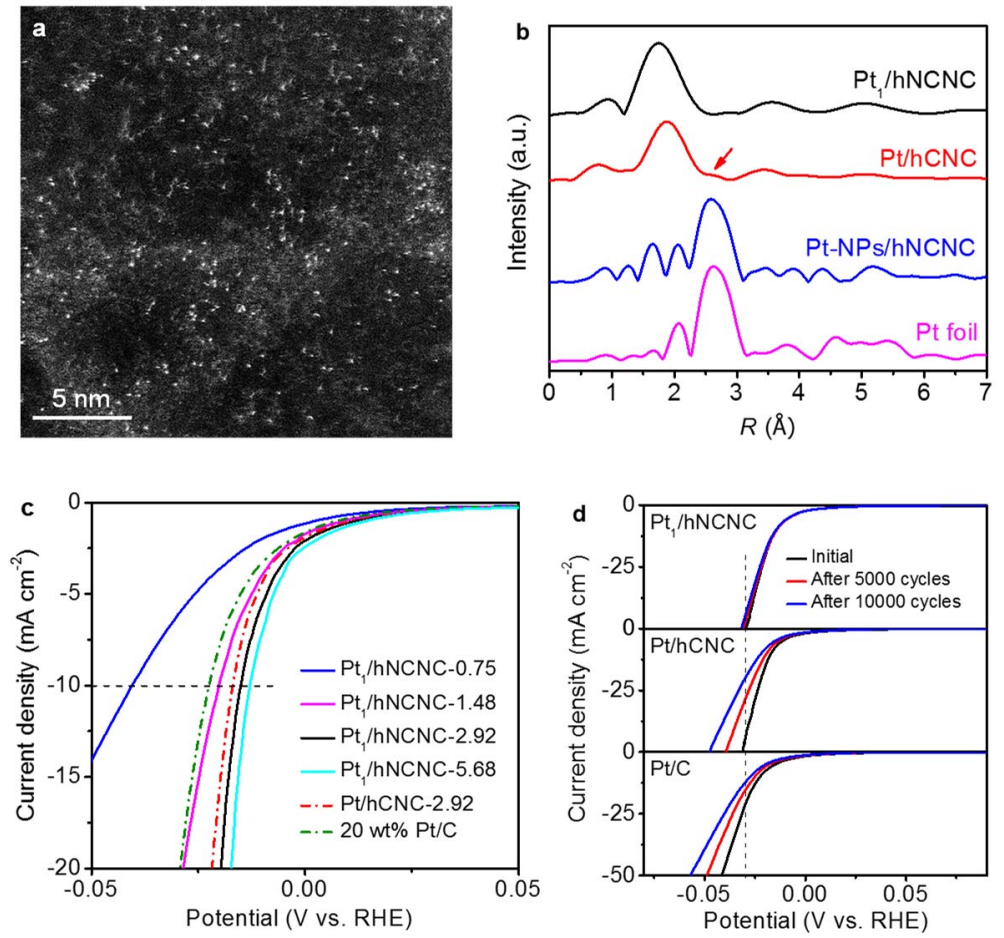

$\mathrm{Pt}_{1} / \mathrm{hNCNC}$ 单位点催化剂的形貌与氢析出性能: (a) HAADF-STEM 照片; (b) EXAFS 图谱, 其中 Pt/hCNC 表示通过 相同方法在未掺杂碳纳米笼上构建的催化剂, Pt-NPs/hNCNC 表示 $\mathrm{hNCNC}$ 负载的 Pt 纳米粒子催化剂, $\mathrm{Pt}_{1} / \mathrm{hNCNC}$ 和

$\mathrm{Pt} / \mathrm{hCNC}$ 中 Pt 载量均为 $2.92 \%$ ( $w$, 质量分数); (c)氢析出线性扫描伏安谱, 其中 $\mathrm{Pt}_{1} / \mathrm{hNCNC}$ 后接数值表示 $\mathrm{Pt}$

载量 $(\%) ;(d)$ 氢析出稳定性, $\mathrm{Pt}_{1} / \mathrm{hNCNC}$ 和 $\mathrm{Pt} / \mathrm{hCNC}$ 中 $\mathrm{Pt}$ 载量均为 $2.92 \%(w), \mathrm{Pt} / \mathrm{C}$ 中 $\mathrm{Pt}$ 载量为 $20 \%(w)$ 。

单位点催化剂(文献中也时常称为单原子催 化剂)是负载型催化剂的极限, 因其最大的原子利 用率、独特的催化性能、均一的配位环境等特点成 为近年来科学研究的前沿热点 ${ }^{1}$ 。金属单原子表面
能高, 容易团聚, 其制备过程通常较为复杂, 因此, 方便地构建稳定性高的单位点催化剂对于开展相 关基础或应用研究均意义重大 ${ }^{2-4}$ 。

近年来, 南京大学胡征教授领衔的能源纳米 
材料物理化学课题组发展了具有自主知识产权的 技术路线, 开发出具有新颖介观结构、高比表面 积、微孔-中孔-大孔共存、便于掺杂调变的碳基纳 米笼新材料, 展现出一系列十分优异的能源转化 及储存性能 ${ }^{5-7}$ 。本项研究中, 他们以氮掺杂碳纳米 笼( $\mathrm{hNCNC}$ )作载体, 利用笼壁上丰富的微孔( 0.6 $\mathrm{nm})$ 易于捕获金属配位阴离子、掺杂氮原子利于针 定金属原子的协同作用, 通过最简单的浸渍-吸附烘干 $\left(70^{\circ} \mathrm{C}\right)$ 过程, 制得了 $\mathrm{M}_{1} / \mathrm{hNCNC}(\mathrm{M}=\mathrm{Pt} 、 \mathrm{Pd}$ 、 $\mathrm{Au} 、 \mathrm{Ir}$ 等)单位点贵金属催化剂。 $\mathrm{Pt}_{1} / \mathrm{hNCNC}$ 催化剂 的Pt载量可高达 $5.68 \%(w$, 质量分数), 在酸性介质 中表现出极低的氢析出过电位、超高的质量活性 和优异的稳定性(见上图)。通过理论和实验研究揭 示了 $\mathrm{Pt}$ 单位点催化剂的形成机制及其优异稳定性 的根源。本项研究揭示的微孔捕获和杂原子针定 的协同作用为方便构建高稳定单位点催化剂提供 了新的思路和方法, 对于促进单位点催化剂的基 础研究和实际应用具有重要价值。相关论文最近 发表于Nature Communications 杂志上 ${ }^{8}$ 。该研究得 到了国家自然科学基金重点项目、科技部纳米重 大研究计划等项目的资助。

\section{References}

(1) Qiao, B.T.; Wang, A. Q.; Yang, X. F.; Allard, L. F.; Jiang, Z.; Cui, Y. T.; Liu, J. Y.; Li, J.; Zhang, T. Nat. Chem. 2011, 3, 634. doi: 10.1038/NCHEM.1095

(2) Yang, X. F.; Wang, A. Q.; Qiao, B. T.; Li, J.; Liu, J. Y.; Zhang, T. Acc. Chem. Res. 2013, 46, 1740. doi: 10.1021/ar300361m

(3) Zhang, H. B.; Liu, G. G.; Shi, L.; Ye, J. H. Adv. Energy Mater. 2018, 8, 1701343. doi: 10.1002/aenm.201701343

(4) Zhu, C. Z.; Fu, S. F.; Shi, Q. R.; Du, Q.; Lin, Y. H. Angew. Chem. Int. Ed. 2017, 56, 13944. doi: 10.1002/anie.201403864

(5) Xie, K.; Qin, X. T.; Wang, X. Z.; Wang, Y. N.; Tao, H. S.; Wu, Q.; Yang, L. J.; Hu, Z. Adv. Mater. 2012, 24, 347. doi: 10.1002/adma.201103872

(6) Wu, Q.; Yang, L. J.; Wang, X. Z.; Hu, Z. Acc. Chem. Res. 2017, 50, 435. doi: $10.1021 /$ acs.accounts.6b00541

(7) Yang, L. J.; Shui, J. L.; Du, L.; Shao, Y. Y.; Liu, J.; Dai, L. M.; Hu, Z. Adv. Mater. 2019, 31, 1804799. doi: 10.1002/adma.201804799

(8) Zhang, Z. Q.; Chen, Y. G.; Zhou, L. Q., Chen, C.; Han, Z.; Zhang, B. S.; Wu, Q.; Yang, L. J.; Du, L. Y.; Bu, Y. F.; et al. Nat. Commun. 2019, 10, 1657. doi: 10.1038/s41467-019-09596-x 